\title{
Traveling ionospheric disturbances in the Weddell Sea Anomaly associated with geomagnetic activity
}

\author{
S. E. Milan, ${ }^{1}$ A. Grocott, ${ }^{1,2}$ S. de Larquier, ${ }^{3}$ M. Lester, ${ }^{1}$ T. K. Yeoman, ${ }^{1}$ \\ M. P. Freeman, ${ }^{4}$ and G. Chisham ${ }^{4}$ \\ Received 30 January 2013; revised 12 August 2013; accepted 12 September 2013; published 3 October 2013.
}

[1] We present observations from the Falkland Islands Super Dual Auroral Radar Network radar of the propagation of HF radio waves via the Weddell Sea Ionospheric Anomaly (WSA), a region of enhanced austral summer nighttime ionospheric electron densities covering the southern Pacific and South Americas region. This anomaly is thought to be produced by uplift of the ionosphere by prevailing equatorward thermospheric winds. Of particular interest are perturbations of the WSA-supported propagation, which suggest that during periods of geomagnetic disturbance, the ionospheric layer can be lowered by several tens of kilometers and subsequently recover over a period of 1 to $2 \mathrm{~h}$. Perturbations can appear singly or as a train of two to three events. We discuss possible causes of the perturbations and conclude that they are associated with equatorward propagating large-scale atmospheric waves produced by magnetospheric energy deposition in the auroral or subauroral ionosphere. Changes in high/ middle latitude electrodynamics during geomagnetic storms may also account for the perturbations, but further modeling is required to fully understand their cause.

Citation: Milan, S. E., A. Grocott, S. de Larquier, M. Lester, T. K. Yeoman, M. P. Freeman, and G. Chisham (2013), Traveling ionospheric disturbances in the Weddell Sea Anomaly associated with geomagnetic activity, J. Geophys. Res. Space Physics, 118, 6608-6617, doi:10.1002/jgra.50566.

\section{Introduction}

[2] In this paper, we investigate two, possibly related, ionospheric phenomena observed in the vicinity of the Antarctic Peninsula, but which are considered to be of significance over a much wider geographical extent. The first of these phenomena is the summer, nighttime enhancement of ionospheric densities above that expected through photoionization; the second phenomenon relates to perturbations of this ionospheric anomaly which appear to be produced by large-scale atmospheric waves launched from the auroral zone during periods of geomagnetic disturbance. Our observations suggest that ionospheric perturbations during the day are usually associated with medium-scale waves (MSWs) but that large-scale waves (LSWs) dominate at night.

[3] Solar photoionization of the neutral atmosphere is the dominant process by which the Earth's ionosphere is formed. As a consequence, the morphology and variability of the ionosphere at any location on the globe are largely controlled by diurnal and seasonal variations in solar illumination and the

\footnotetext{
${ }^{1}$ Department of Physics and Astronomy, University of Leicester, Leicester, UK.

${ }^{2}$ National Institute of Polar Research, Tokyo, Japan.

${ }^{3}$ SuperDARN HF Radar Group, Virginia Tech, Blacksburg, Virginia, USA.

${ }^{4}$ British Antarctic Survey, Cambridge, UK.

Corresponding author: S. Milan, Department of Physics and Astronomy, University of Leicester, University Rd., Leicester LE1 7RH, UK. (steve.milan@ion.le.ac.uk)

(C)2013. American Geophysical Union. All Rights Reserved. 2169-9380/13/10.1002/jgra.50566
}

solar cycle modulation of the flux of EUV photons. The resulting ionospheric plasma density is a balance between the ionization rate, which maximizes at the subsolar point, and loss processes; these loss processes are usually dependent on the density and composition of the background neutral atmosphere and tend to be more important at lower altitudes. Other processes act to perturb this pattern, including impact ionization by auroral precipitation and plasma transport processes. The lifting of ionospheric plasma to higher altitudes, by electrodynamic processes or the blowing of thermospheric winds across inclined magnetic field lines, can reduce loss rates and hence sustain higher ionospheric densities in certain geographical and local time regions where photoionization is not expected to be significant. Currently, little is known about the exact formation mechanisms that give rise to these regions of enhanced density or "ionospheric anomalies".

[4] The Northern Hemisphere Summer Evening Anomaly, as one of the ionospheric anomalies, was recently observed by de Larquier et al. [2011] in HF radio-sounding experiments with the Blackstone radar, part of the Super Dual Auroral Radar Network (SuperDARN) [Chisham et al., 2007]. This anomaly was identified as a sustainment of the propagation of HF radio waves in the ionosphere for several hours beyond sunset (marking the cessation of photoionization) in the Northern Hemisphere summer months April to September. This propagation was shown to be supported by high postsunset ionospheric plasma densities, which were observed with the nearby Millstone Hill incoherent scatter radar. de Larquier et al. [2011] demonstrated that ray tracing through the International Reference Ionosphere (IRI) [Bilitza, 2001] 


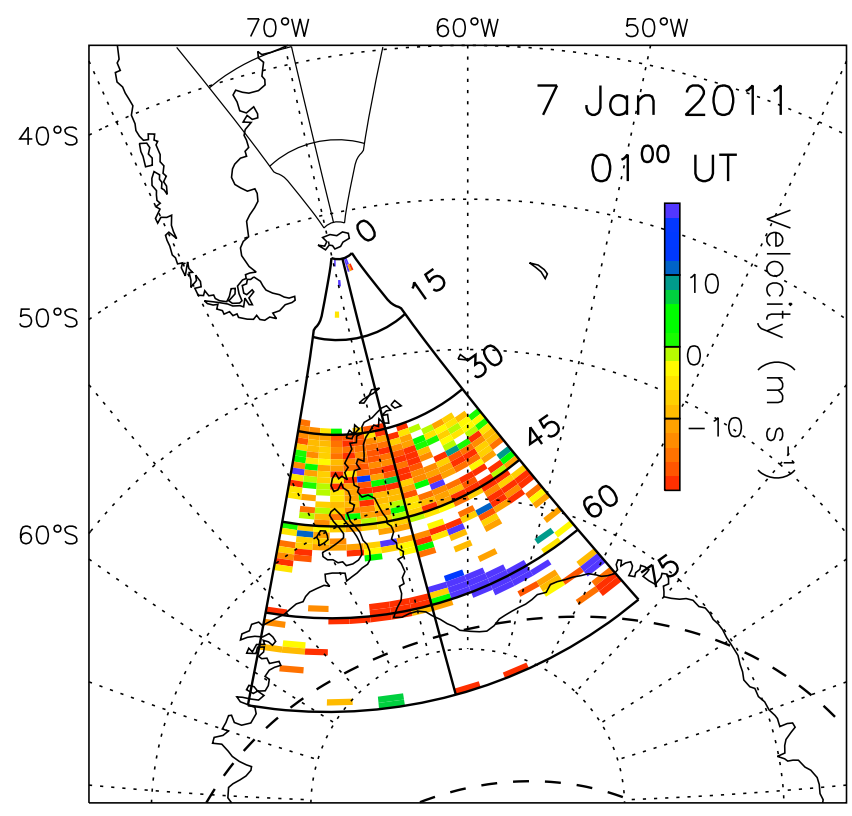

Figure 1. The location of the field of view of the Falkland Islands SuperDARN radar, comprising 16 beams and 75 range gates. The locations of echoes observed during the scan starting 01 UT on 7 January 2011 are shown, color coded by Doppler velocity. Echoes observed in range gates 30 to 50 are ground backscatter, and echoes from near gate 60 are of ionospheric origin. The poleward and equatorward edges of the auroral zone for $K p=3$ are indicated by dashed curves.

predicted the occurrence of this propagation mode, indicating that the IRI captured this ionospheric feature. In addition, modeling of the interaction of prevailing thermospheric winds and the geomagnetic field supported the idea that this anomaly is produced by ionospheric uplift.

[5] As reported by Horvath and Essex [2003] and Horvath [2006], a corresponding ionospheric anomaly occurs in the Southern Hemisphere in the region of the Antarctic Peninsula during austral summer, known as the Weddell Sea Anomaly (WSA), which is also thought to be maintained by ionospheric uplift produced by equatorward neutral winds [e.g., Chen et al., 2011]. The Falkland Islands SuperDARN radar is ideally placed to investigate the occurrence of this anomaly. In this paper, we show that the anomaly is indeed observed, we study short time scale perturbations of the anomaly which we associate with traveling ionospheric disturbances, and we demonstrate their dependence on periods of geomagnetic activity.

\section{The Falkland Islands SuperDARN Radar}

[6] The Falkland Islands Super Dual Auroral Radar Network high-frequency coherent scatter radar (hereafter FIR) was built to the standard SuperDARN specification [Greenwald et al., 1995] and operated between February 2010 and October 2011. Its field of view looked south from the Falkland Islands over the Antarctic peninsula, as indicated in Figure 1. The radar scanned through 16 beam directions, separated by $3.24^{\circ}$, every minute, transmitting at a frequency of $12 \mathrm{MHz}$. Backscattered signals were gated into
75 range gates, each of length $45 \mathrm{~km}$, with $180 \mathrm{~km}$ to the first gate. The radar transmitted a seven-pulse sequence that allowed the power, spectral width, and Doppler shift of backscattered echoes to be determined. As an example, Figure 1 shows the locations of echoes observed during the scan starting at 01:00 UT on 7 January 2011. The echoes are color coded by the Doppler shift imposed by the movement of the ionospheric scatters or motions of the ionosphere; positive (blue) Doppler velocities indicate movement toward the radar.

[7] As with all SuperDARN radars, some echoes are caused by coherent scatter from electron density irregularities in the ionosphere (called ionospheric scatter), whereas others are associated with obliquely propagating radio waves refracted by the ionosphere back to the ground, thence scattered back along the original raypath and received by the radar (called ground scatter). Such ground scatter is identified by virtue of having a low imposed Doppler shift, typically $\left|V_{\text {Dopp }}\right|<50 \mathrm{~m} \mathrm{~s}^{-1}$, and a low spectral width, $\Delta V_{\text {Dopp }}<25 \mathrm{~m} \mathrm{~s}^{-1}$. The occurrence, location, and Doppler shift of ground scatter echoes give information about the ionospheric electron density and motions of ionospheric layers within the field of view of the radar, and for this reason, these are the focus of the present study. In the example shown in Figure 1, the echoes between range gates 30 and 50 are of low velocity and are expected to arise as a consequence of ground scatter. Echoes in a narrow latitudinal band near range gate 60 have much higher Doppler velocities (saturated on the color scale) and are ionospheric scatter associated with a subauroral ion drift (SAID) event, as will be discussed in more detail in section 4.1 .

[8] The range from which backscatter originates is determined by the time-of-flight of radar pulses, assuming propagation occurred at the speed of light. When determining the "ground range" or geographic location of the scatterers, corrections are made to take into account the slowing of the propagation in the ionosphere and the vertical component of the ray propagation. In this study, the exact geographic location of the ground scatters is not important and we are interested in the propagation of the radar signals through the ionosphere. Hence, we use the uncorrected "radar range" or "slant range", demarcated in radar gate number, where a particular gate $n$ is located at a radar range of $180+45 n \mathrm{~km}$. We should also note that in the case of ground backscatter, when echoes are detected at a particular range, the distance to the point of reflection in the ionosphere is approximately half this distance. In Figure 1, the leading edge of the ground scatter is observed near range gate $30(1530 \mathrm{~km})$ and the reflection point in the ionosphere will be near a range of $765 \mathrm{~km}$, corresponding to the location of range gate 13 .

[9] Although the antennas of SuperDARN radars are designed to radiate most power in the forward direction, some power escapes from the rear and this can also be backscattered to the radar. Radars equipped with an interferometer array can distinguish between backscatter originating in the forward and rear lobes of the antenna patterns [Milan et al., 1997a]. Unfortunately, FIR did not have an interferometer array so; as will be discussed in section 4, it must be borne in mind that the ground scatter could be reflected near range gate 15 in the field of view of the rear lobe, indicated by thin lines in Figure 1. 

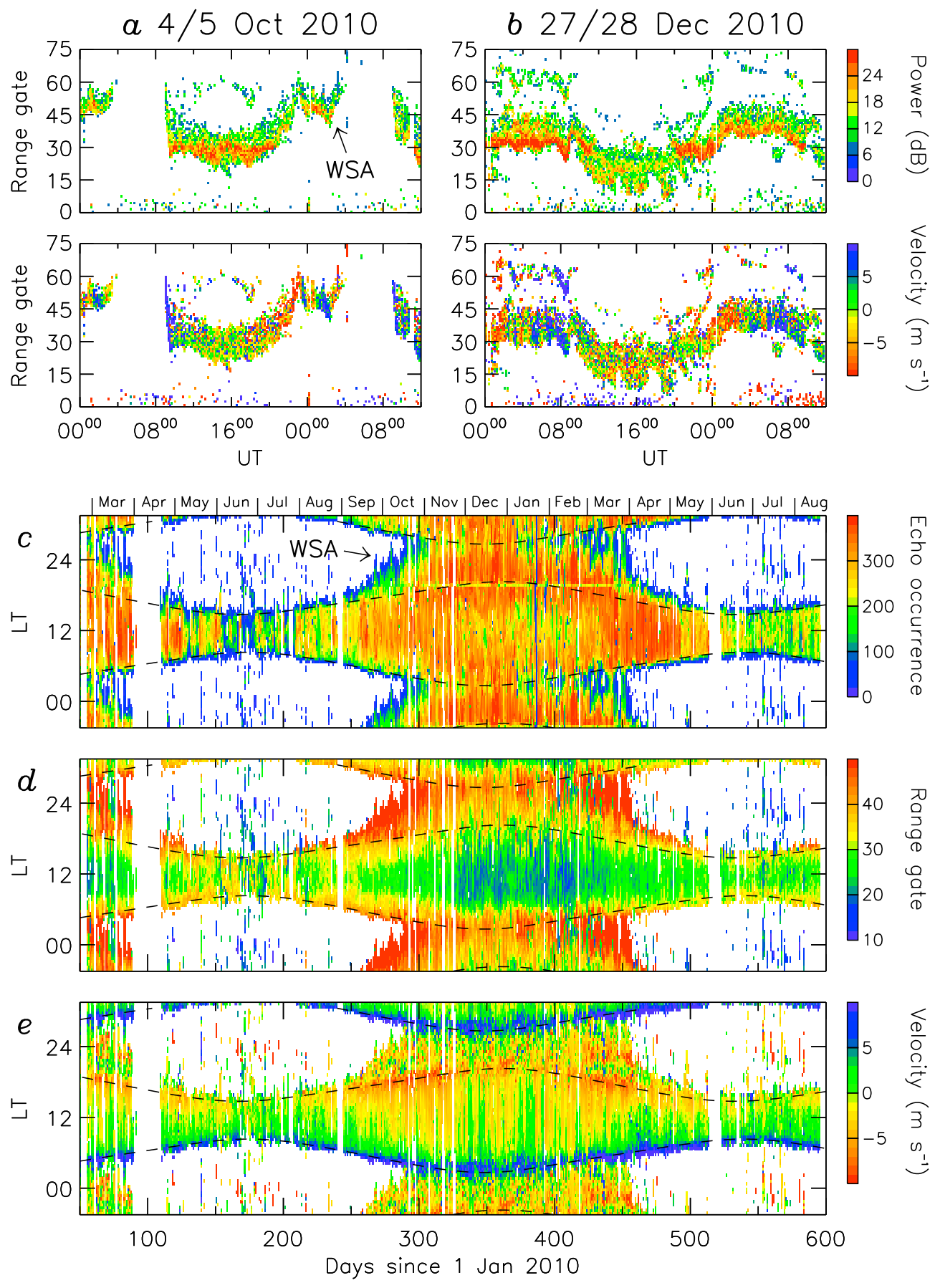

Figure 2. ( $a$ and $b$ ) Range-time plots of ground backscatter occurrence along a central beam of the radar (beam 7), color coded according to echo power (top) and Doppler velocity (bottom) for 4/5 October and 27/ 28 December 2010, respectively. (c to e) The local time occurrence of backscatter for the period March 2010 to August 2011, color coded by (Figure 2c) echo count, (Figure 2d) median range gate, and (Figure 2e) median Doppler velocity. Dashed lines indicate times at which the solar zenith angle is $90^{\circ}$ at the ionospheric reflection point.

\section{Observations}

\subsection{Morphology of Weddell Sea Anomaly Echoes}

[10] Figure 2a shows range-time plots of backscatter power and Doppler shift observed along the central beam (beam 7) of the FIR field of view from 00 UT on 4 October 2010 to $12 \mathrm{UT}$ on the following day (a total of $36 \mathrm{~h}$ ). Figure $2 \mathrm{~b}$ shows the corresponding data for 27/28 December 2010. In the first 10 range gates or so, sporadic echoes are backscattered from ablating meteors [e.g., Hall et al., 1997]. Beyond this, the main region of echoes is ground scatter. The range to the leading edge of this backscatter, known as the skip distance, is the nearest range to which the ionosphere can refract a given radio frequency, and depends on the maximum 
electron density in the $F$ region, as will be discussed in more detail in section 4.2. As described by Milan et al. [1997b] and de Larquier et al. [2011], the expected diurnal variation in ground backscatter observed by a SuperDARN radar is a U-shaped region appearing roughly symmetrically about local noon ( $\sim 16$ UT at FIR). Considering Figure 2a first, backscatter appears at farther ranges at sunrise ( 09 UT) and moves to shorter ranges as the ionospheric density increases; after midday, the backscatter retreats to farther ranges as the ionospheric density decreases. Within this region of backscatter, Doppler velocities are seen to be largely positive (negative) as the scatter moves toward (away from) the radar in the prenoon (postnoon) sector.

[11] The backscatter is expected to disappear shortly after sunset ( 23 UT) as the ionospheric density becomes insufficient to support propagation to the ground any longer. However, a secondary region of backscatter is observed postsunset (23-05 UT), and it is this backscatter that is attributed to propagation via the Weddell Sea Anomaly (see de Larquier et al. [2011] for examples of propagation via the corresponding Northern Hemisphere Summer Evening Anomaly). In the second example, taken just after austral summer solstice (Figure 2b), the observations are similar, but now propagation is supported throughout the night by the WSA.

[12] Short time scale variations in skip distance and Doppler shift, for instance between 00 and 08 UT in Figure 2b, are associated with motions of the ionosphere due to propagating atmospheric waves [e.g., Samson et al., 1990]. It is such changes in WSA scatter that form the focus of this paper.

\subsection{Seasonal Variation in Ground Backscatter and WSA Echoes}

[13] Figures 2c-2e summarize the ground scatter observations from FIR for the period mid-February 2010 to midAugust 2011. Each day of observations is divided into 48 half-hour bins; within each bin, we determine the number of ground scatter echoes received (Figure 2c), the median range at which these echoes are observed (Figure 2d), and the median Doppler shift of the echoes (Figure 2e). This information is displayed with local time varying vertically, while day number since 1 January 2010 varies horizontally. Vertical bands of no data, the most significant being around day 100 , are due to breaks in the operation of the radar. Superimposed on the figures is the variation in the time of sunrise and sunset (solar zenith angle, SZA, of $90^{\circ}$ ) at the approximate path midpoint of the scatter. The majority of echoes occur when $\mathrm{SZA}<90^{\circ}$, which is when the ionosphere is illuminated (Figure 2c). In fact, scatter is observed a short time before sunrise and shortly after sunset as the ionosphere continues to be illuminated at $\mathrm{SZA} \approx 100^{\circ}$ due to the curvature of the Earth. During this period of solar illumination, the scatter is at far ranges near sunrise and sunset, nearing the radar near noon (Figure 2d), as described in section 3.1. Associated with these motions of the scatter, the Doppler shifts observed tend to be positive around sunrise and negative around sunset (Figure 2e), as described in section 3.1.

[14] Between the months of September and April (days 250 to 500), echoes are seen to persist after sunset (Figure 2c), and it is these echoes that are attributed to the
WSA. In September and April, the echoes persist only for a few hours after sunset (cf. Figure 2a); between November and March, the echoes persist all night until sunrise (cf. Figure $2 \mathrm{~b}$ ). These echoes tend to be located at relatively far ranges (Figure 2d), consistent with the two examples shown. Also, the Doppler shifts observed within these echoes are variable and unordered (Figure 2e), as can also be seen in the examples. It is the variability of these Doppler shifts that will be discussed next.

\subsection{WSA Echo Perturbations and Dependence on Geomagnetic Activity}

[15] Figure 3 shows four examples of WSA echoes, focusing on the nighttime period between sunset and sunrise. The first example (Figure 3a) shows a period during which the range and Doppler shifts of the echoes are relatively uniform all night. Higher Doppler shifts around 00 and $08 \mathrm{UT}$ are associated with ionospheric changes at sunset and sunrise, respectively. Figure 3a (inset) focuses on a period of observations during which quasiperiodic fingers of ground backscatter appear to move away from the radar. These are the signatures of small-scale traveling ionospheric disturbances that produce focusing and defocusing of the radar signals [e.g., Samson et al., 1990; MacDougall et al., 2001].

[16] The other examples show periods when large perturbations are observed in both range and Doppler shift. In general, these appear as pairs of toward/away motions of the scatter and corresponding positive/negative excursions in the Doppler shifts, lasting approximately $2 \mathrm{~h}$ (indicated by arrows). Sometimes these are isolated (Figure 3b: 08 UT), whereas during other examples, they occur in trains of several perturbations (Figure 3c: 01, 03, and $05 \mathrm{UT}$; Figure 3d: 00, 02, and 04 UT). The nature of these perturbations will be examined in detail below. First, we investigate their occurrence.

[17] To objectively identify those periods with WSA perturbations, we looked for instances of enhanced variability in the WSA echo Doppler shifts. For the period day 250 to day 500, we identified nighttime echoes by requiring that $\mathrm{SZA}>95^{\circ}$. We included observations from all radar beams but discounted the first 10 range gates, which are dominated by meteor echoes, and ranges beyond gate 55 which can contain subauroral ionospheric backscatter (e.g., see Figure 1). For each night, we formed an occurrence distribution of the observed Doppler shifts, of which two examples are shown in Figure 4 (bottom). These occurrence distributions were roughly Gaussian in shape and usually centered near $0 \mathrm{~m} \mathrm{~s}^{-1}$ Doppler shift (see 23 January 2011, example A). During periods of perturbation activity, these distributions broadened and it is this signature which we use to identify perturbations (see 11 March 2011, example B). We defined the width of the distributions as the difference between the upper and lower deciles of the distributions (vertical dotted lines in the example distributions), and this is plotted as the middle trace in Figure 4 (top). The lower trace of Figure 4 (top) indicates the number of echoes in each occurrence distribution; we set a minimum threshold of 5000 echoes for the determination of a distribution width. The distribution width tended to be close to $20 \mathrm{~m} \mathrm{~s}^{-1}$, but there are clear peaks in the width when this rises as high as $40 \mathrm{~m} \mathrm{~s}^{-1}$. 

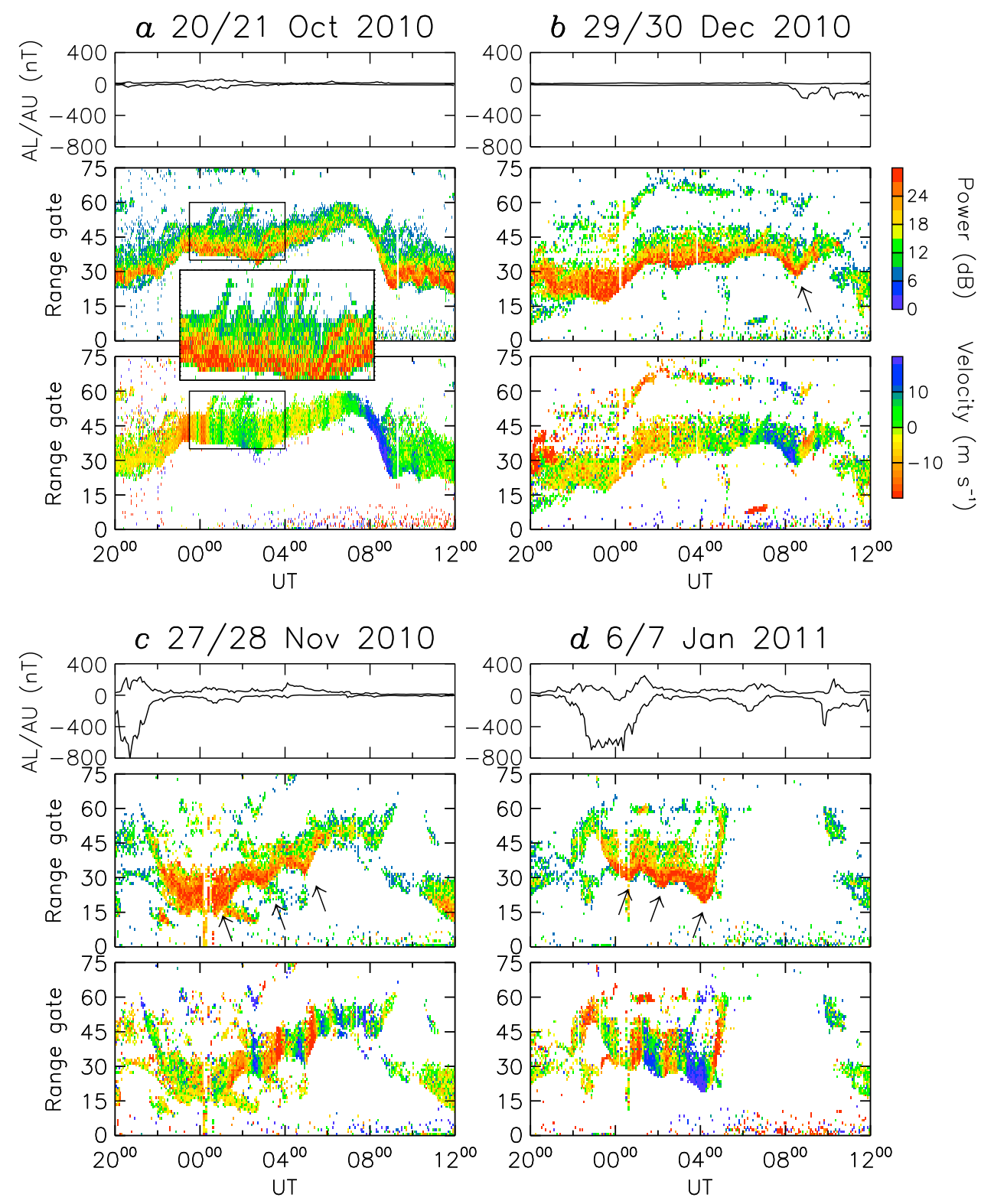

Figure 3. $(\mathrm{a}-\mathrm{d})$ Four examples of nighttime ground backscatter supported by the Weddell Sea Anomaly. (top) The $A U$ and $A L$ indices for the corresponding times. (middle and bottom) Range-time plots of ground backscatter occurrence color coded by backscatter power and Doppler velocity, respectively. Instances of perturbations of the WSA are highlighted by arrows.

[18] This method clearly identified isolated nights of enhanced perturbation activity (indeed, the peaks labeled $\mathrm{c}$ and $\mathrm{d}$ correspond to the examples shown in Figures $3 \mathrm{c}$ and $3 \mathrm{~d}$, respectively). To test to see if there is an association between perturbation activity and geomagnetic activity, we have plotted the $S Y M-H$ index as the upper trace in Figure 4 (top). SYM- $H$ measures perturbations in the north-south component of the magnetic field in the equatorial region at the Earth's surface. Positive excursions in SYM- $H$ are associated with enhancements in the dayside magnetopause currents due to compression of the magnetosphere by enhanced solar wind ram pressure. Negative excursions are produced by the magnetic field associated with an enhanced ring current. A positive/ negative excursion in $S Y M-H$ is characteristic of the arrival of a solar wind shock at the Earth and enhanced energy input to the magnetosphere through solar wind-magnetosphere coupling, known as a geomagnetic storm. Such periods are usually associated with enhanced energy input into the auroral zone atmosphere. Several of our periods of enhanced WSA perturbations do indeed appear to correspond to the occurrence of geomagnetic storms; the clearest examples have been indicated with vertical dotted lines in Figure 4. One of the largest perturbations appears on day 257 of 2010 but corresponds to only a modest storm signature in SYM-H. However, the number of WSA echoes that day is very low and we consider the distribution to be susceptible to noise in the observations.

\subsection{Analysis of Backscatter Perturbations}

[19] The Doppler shifts of the WSA echoes from the night of 6/7 January 2011 are examined in detail in Figure 5 

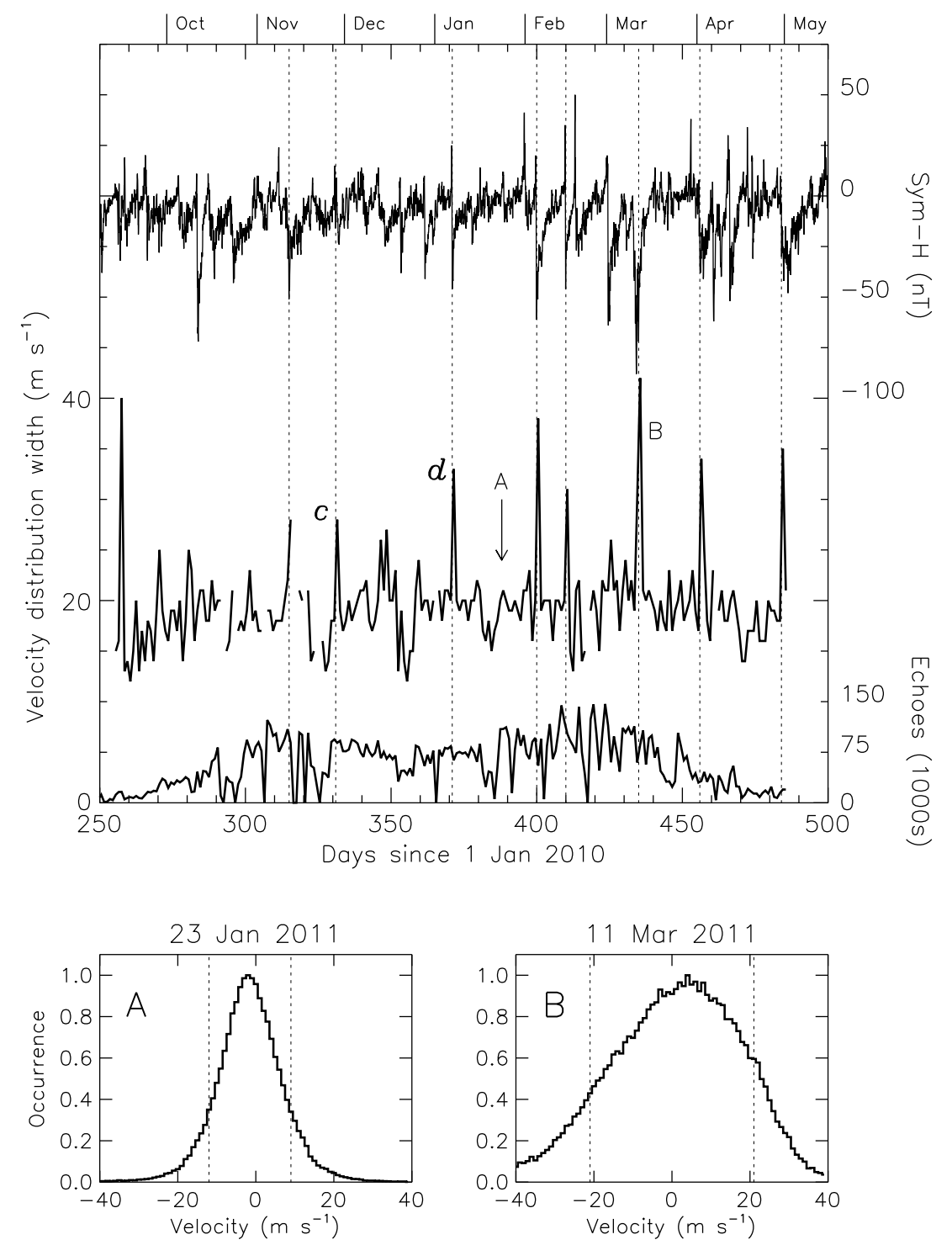

Figure 4. A comparison of the occurrence of perturbations of the WSA for the period September 2010 to May 2011. (top) The $S Y M-H$ index with characteristic bipolar signatures indicating the occurrence of geomagnetic storms (upper trace); the width of the distribution of Doppler velocity in WSA backscatter for each night of observation, in which elevated widths indicate perturbations (middle trace); and the number of echoes in each distribution (lower trace). A width is only calculated when more than 5000 echoes contributed to the distribution. (bottom) Two examples of velocity occurrence distributions from 23 January (A) and 11 March 2011 (B).

(see also Figure 3d; Figure 1 is a scan from 01 UT on 7 January 2011). Figure 5a is a range-time-velocity plot from beam 7 of the radar. The Doppler shifts range in general between -20 and $20 \mathrm{~m} \mathrm{~s}^{-1}$. Within the main region of backscatter, these velocity perturbations are largely independent of range, suggesting that the vertical ionospheric motions that give rise to these Doppler shifts occur across a significant geographic area. To visualize the velocity changes more clearly, we have selected gate 33 of beam 7 (indicated as a horizontal dashed line) and reproduced the Doppler shifts from it as a line plot in Figure 5b.

[20] We note also that in addition to a variation in Doppler shift, the leading edge (nearest range) of the backscatter has a wave-like nature. When the Doppler shift is negative, the leading edge retreats to farther ranges and approaches the radar again when the Doppler shifts are positive. A more detailed analysis of these observations takes place in section 4.2.

\section{Discussion}

\subsection{WSA Occurrence and Perturbations}

[21] The Weddell Sea Ionospheric Anomaly covers a relatively large geographic region, encompassing the Antarctic Peninsula, the southern areas of South America, stretching westward to the mid-Pacific, occurring predominantly in local summer months [e.g., Chen et al., 2011]. Our observations with the Falkland Islands radar are hence of the eastern portion of the WSA. In common with the Northern Hemisphere Summer Evening Anomaly, the WSA is thought to be maintained by equatorward thermospheric winds which 

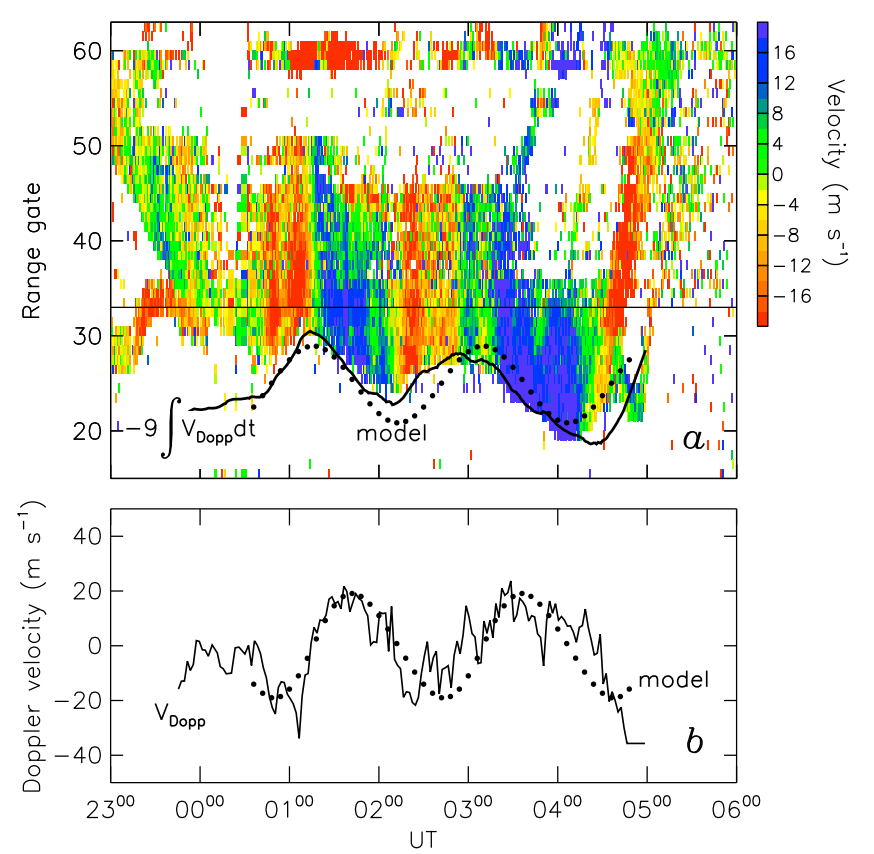

Figure 5. (a) Range-time plot of echo occurrence on the night of the 6/7 January 2011, color coded by Doppler velocity. Range gate 33 is indicated by a horizontal dashed line. The solid curve represents the integrated Doppler velocity from range gate 33 multiplied by a factor of -9 , positioned to coincide with the leading edge of the backscatter. The dots indicate a model of the leading edge motion (see text for details). (b) The Doppler velocity from range gate $33, V_{\text {Dopp }}$. Dots are a model variation (see text for details).

push the ionospheric plasma up the inclined geomagnetic field to altitudes where recombination processes are slow [de Larquier et al., 2011; Chen et al., 2011]. The observation of a propagation mode supported by the WSA during the months September to March is consistent with the expected annual variation from the modeling of Chen et al. [2011].

[22] Small-scale perturbations of the WSA are observed (Figure 3a), associated with atmospheric gravity waves, which are a common feature of SuperDARN observations all over the globe [e.g., Samson et al., 1990]. In this study, however, we focus on larger-scale perturbations of the WSA (Figures 3b-3d) which appear linked to periods of enhanced geomagnetic activity.

[23] We note that in the case of the small-scale perturbations (Figure 3a), the backscatter features appear to propagate away from the radar suggesting poleward propagation, whereas much previous work [e.g., Samson et al., 1990] has suggested that such waves are often excited at auroral latitudes and propagate equatorward. It is possible that this backscatter originates behind the radar (see section 2), in which case the waves are indeed traveling equatorward. Alternatively, these waves originate not from the auroral zone but some other source (e.g., the orography of South America) [e.g., Wu and Jiang, 2002; Hocke et al., 2002]. We mention this to indicate that there is some ambiguity in the location of the ionospheric reflection point of the WSA ground backscatter (near gate 15, either in front of or behind the radar). However, this ambiguity does not affect the rest of our discussion as both possible reflection points are located within the Weddell Sea anomaly.

[24] Figure 4 shows the association between perturbed Doppler shifts observed by FIR in WSA backscatter and geomagnetic storm signatures in the $S Y M-H$ index. In addition, Figures $3 \mathrm{a}-3 \mathrm{~d}$ (top) show the $A U$ and $A L$ indices, which measure the strength of currents flowing in the Northern Hemisphere auroral electrojets (which should also be a good indicator of electrojet activity in the Southern Hemisphere). Enhanced electrojet activity is an indicator of energy deposition in the auroral zone, especially when there are significant negative excursions of the $A L$ index. Little geomagnetic activity occurs in Figure 3a; the WSA perturbation of Figure $3 \mathrm{~b}$ occurs contemporaneously with a relatively weak enhancement of activity; the trains of perturbations in Figures $3 \mathrm{c}$ and $3 \mathrm{~d}$ occur in the hours following periods of significant activity.

[25] In addition, there is a region of ionospheric backscatter in range gates 57 to 61 in Figure 1, just equatorward of the lower latitude edge of the auroral zone (dashed lines show the poleward and equatorward edges of the Feldstein oval [Holzworth and Meng, 1975] for $K p=3$ ). Doppler velocities toward the radar in the western portion of the field of view and velocities away from the radar in the eastern portion indicate rapid sunward azimuthal flows in a narrow latitudinal region in the premidnight sector. This is the signature of a subauroral ion drift (SAID), often observed during geomagnetic storms [e.g., Grocott et al., 2011], indicating again that the 6/7 January 2011 period is associated with a period of enhanced energy deposition in the auroral zone atmosphere.

\subsection{Propagation Considerations}

[26] Section 3.4 indicated that the Doppler shifts observed in the WSA echoes and the motion of the leading edge of the backscatter are related. In this section, we investigate the implications of this relationship for the nature of the perturbations. Returning to Figure 5, the Doppler shifts imply that the ionosphere is moving and we compare this with the motion of the leading edge of the backscatter region, the skip distance $S$. We have integrated the Doppler shift from Figure $5 \mathrm{~b}$ with time to determine the implied change in range, $\Delta R$, and superimposed this on Figure 5a (black curve), positioned to match the range of the leading edge of the backscatter. We see an excellent agreement between motions implied by the Doppler shifts and the changing position of the leading edge; however, to achieve this fit, we have had to apply a scaling factor of -9 to $\Delta R$, that is, $\Delta S \approx-9 \Delta R$. To understand the implications of this scaling factor, we consider the propagation conditions that allow the radar to detect backscatter from the ground at particular ranges.

[27] For simplicity, we will assume a flat Earth and straight-line propagation, in which case all altitudes will be virtual altitudes (Breit and Tuve's theorem and Martyn's "equivalent path" theorem) [Davies, 1990], as indicated in Figure 6. This figure considers the changes that occur for two example raypaths for an increase in the altitude of the reflecting layer.

[28] The range of the leading edge of the backscatter is determined by the level of refraction of the radar signal in the ionosphere, which in turn is dependent on the electron density profile of the ionosphere. A vertically propagating HF radio 

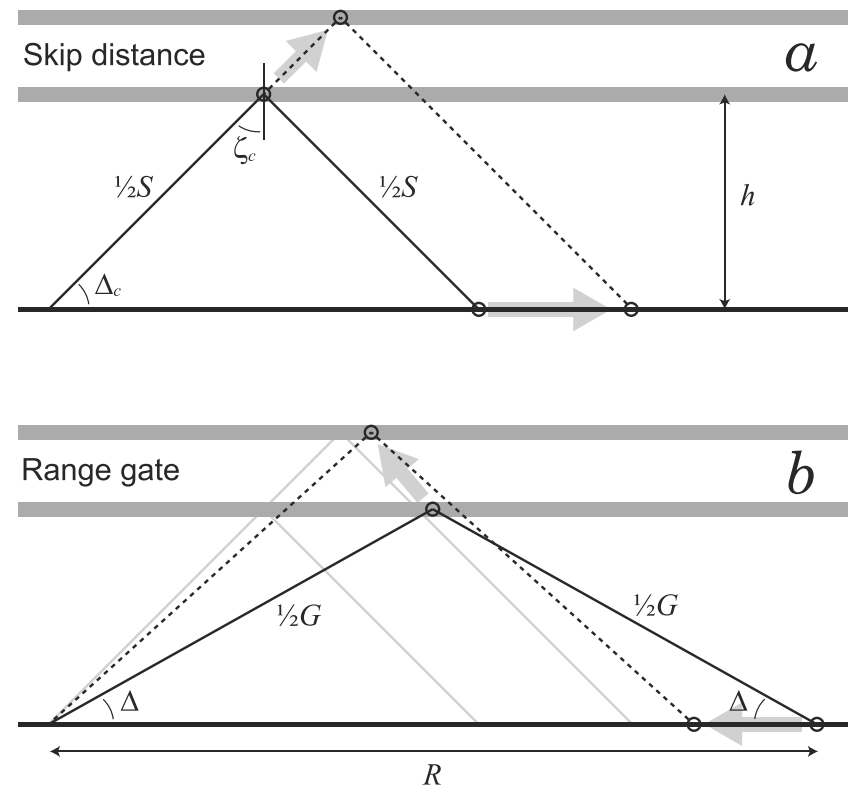

Figure 6. (a) A schematic of the variation in the slant range, $S$, of a radio wave raypath constituting the leading edge of the ground backscatter if the reflecting ionospheric layer increases in altitude, $h$. The full line indicates the ray reflected at the lower height, and the dashed line indicates the ray after the layer has risen. $\Delta_{c}$ and $\zeta_{c}$ indicate the critical elevation and incidence angles, respectively. At greater $\Delta_{c}$ (lower $\zeta_{c}$ ), the ray penetrates the ionosphere and is not reflected to the ground. (b) A corresponding schematic of the variation of the ground range, $R$, of a raypath of fixed slant range, $G$, for the same change in $h$.

wave of frequency $f$ is reflected back to the ground at a height where the ionospheric plasma frequency, $f_{p}$, equals $f$,

$$
f_{p}^{2}=\frac{N_{e} e^{2}}{4 \pi^{2} \varepsilon_{0} m_{e}},
$$

where $N_{e}$ is the electron density, $m_{e}$ is the electron mass, $e$ is the electronic charge, and $\varepsilon_{0}$ is the permittivity of free space. If $f$ exceeds the maximum value of $f_{p}$ in the ionosphere, usually referred to as the critical frequency $f_{c}$, the radio wave propagates into space. For a ray propagating obliquely with takeoff (elevation) angle $\Delta$, such that it is incident on the ionosphere at an angle of $\zeta$ to the vertical, a similar situation holds, except that "reflection" to the ground occurs for angles $\zeta$ such that

$$
f_{c} \sec \zeta>f
$$

known as the "secant law" [Davies, 1990], a consequence of Snell's law. For our flat Earth approximation, $\zeta=90^{\circ}-\Delta$.

[29] The minimum range at which radio waves reach the ground, for which the limiting or critical angle of incidence $\zeta_{c}$ is given by $\sec \zeta_{c}=f / f_{c}$ (with a corresponding $\Delta_{c}$ ), is the leading edge of the ground backscatter region (Figure 6a). Usually, the ground range of the leading edge is known as the skip distance; for the purposes of this paper, we will use the term skip distance to mean the slant range of the leading edge, $S$, as this is the range determined by the radar. We assume a fixed $f_{c}$ (i.e., fixed $\zeta_{c}$ ) and consider changes in the virtual height of the reflecting layer, $h$. Figure 6a shows the paths of two rays with fixed elevation angle $\Delta_{c}$ reflecting from an ionospheric layer at two different heights. The path length of these rays, $S$, depends only on $h$ and is given by

$$
S=2 h \sec \zeta_{c}=2 h \frac{f}{f_{c}}
$$

[30] As $h$ increases, so does $S$. The speed at which the skip distance increases is

$$
V_{\text {skip }}=\frac{\mathrm{d} S}{\mathrm{~d} t}=2 \frac{f}{f_{c}} \frac{\mathrm{d} h}{\mathrm{~d} t} .
$$

[31] We now consider a ray of fixed path length $G(>S)$ which corresponds to a particular radar range gate in which ground backscatter is observed, reflecting at two different heights (Figure 6b). (We assume a thin ionospheric layer, i.e., one in which changes in $\zeta$ do not significantly affect the height of reflection.) The ground range of this ray, $R$, decreases as $h$ increases:

$$
R=\sqrt{G^{2}-4 h^{2}}
$$

[32] To the radar, it will appear that the surface roughness features from which it is scattering move away as the reflection height increases, and this imposes a Doppler shift on the backscattered signal. That Doppler shift corresponds to a velocity $V_{\text {Dopp }}$ given by

$$
V_{\text {Dopp }}=\cos \Delta \frac{\mathrm{d} R}{\mathrm{~d} t}=\cos \Delta \frac{\mathrm{d} R}{\mathrm{~d} h} \frac{\mathrm{d} h}{\mathrm{~d} t} .
$$

[33] As $\cos \Delta=R / G$ and $\mathrm{d} R / \mathrm{d} h=-4 h / R$, this becomes

$$
V_{\text {Dopp }}=-\frac{4 h}{G} \frac{\mathrm{d} h}{\mathrm{~d} t}
$$

[34] From equations (4) and 7, we see that if $h$ is increasing, $V_{\text {skip }}>0$ and $V_{\text {Dopp }}<0$, and vice versa for decreasing $h$, which is the relationship observed in our perturbations: as ground scatter moves toward the radar the Doppler shifts are positive and vice versa. Combining equations (3), (4), and 7, we find the ratio between $V_{\text {skip }}$ and $V_{\text {Dopp }}$ to be

$$
\frac{V_{\text {skip }}}{V_{\text {Dopp }}}=-\frac{G}{2 h} \frac{f}{f_{c}}=-\frac{G S}{4 h^{2}} .
$$

[35] We note that the leading edge of the scatter is located on average near range gate 25 , giving $S \approx 1300 \mathrm{~km}$ (i.e., $180+25 \times 45)$. We evaluated the Doppler shifts in range gate 33 , corresponding to $G=1700 \mathrm{~km}$. From this, using the ratio of -9 found in the fitting in section 3.4, we estimate $h \approx 250 \mathrm{~km}$. From equation (3), we can use $S$ and $h$ to estimate that the ratio $f / f_{c} \approx 2.6$. The radar operating frequency is $12 \mathrm{MHz}$, suggesting that $f_{c} \approx 4.6 \mathrm{MHz}$, giving from equation (1) $N_{\text {emax }} \approx 2.6 \times 10^{11} \mathrm{~m}^{-3}$, similar to values predicted by the IRI [Bilitza, 2001].

[36] As noted in section 3.3, perturbations appear individually or as trains of toward/away motions of the skip distance. 
For the sake of simplicity, we assume that the train of perturbations highlighted in Figure 4 can be treated as a sinusoidal variation in $h$ about a height $h_{0}$ with amplitude $\Delta h$, such that

$$
\begin{aligned}
& h=h_{0}+\Delta h \cos \omega t, \\
& \frac{\mathrm{d} h}{\mathrm{~d} t}=-\omega \Delta h \sin \omega t,
\end{aligned}
$$

and show that $h_{0}=250 \mathrm{~km}, \Delta h=35 \mathrm{~km}$, and $\omega=2 \pi / T$, where $T=1.9 \mathrm{~h}$ is roughly consistent with the observations. Equations (3), (7), (9), and 10 can then be used to find $S$ and $V_{\text {Dopp. }}$. These are superimposed in Figure 5 as dotted lines, and we find a tolerable correspondence with the observations, suggesting that upward and downward motions of the reflecting layer can explain the nature of the perturbations. If the perturbations are indeed formed from individual toward/away motions of the skip distance, this modeling suggests that these correspond to downward/upward motions of the ionosphere.

\subsection{Possible Causes of WSA Perturbations}

[37] Our observations suggest that significant perturbations of the Weddell Sea Anomaly can occur as pairs of downward/upward motions of the ionosphere, on a time scale of 1-2 h. Trains of such perturbations can be observed, appearing as a wave-like motion. Such perturbations occur at times of enhanced geomagnetic activity, when energy is deposited in the high latitude ionosphere. Heating associated with energy deposition might be expected to enhance the equatorward neutral wind that supports the WSA. However, this would produce an uplift of the ionosphere, inconsistent with the observations. A pulse of poleward neutral wind would push the ionosphere downward along the inclined magnetic field, but the cause of such a poleward wind, associated with heating at high latitudes, is difficult to envisage.

[38] Another possible cause is electrodynamic in nature: A westward electric field perturbation coupled with the inclined magnetic field would produce plasma drifts with a vertical component pointing downward, which could account for the lowering of the ionospheric layer. The exact reason for such an electric field perturbation is uncertain, but would be associated with the electrodynamics of the geomagnetic storms with which the perturbations are linked. Figure 5 provides evidence that contradicts this mechanism, however. Between 00 and 04 UT, near range gate 60, ionospheric scatter is observed, which corresponds to the SAID discussed in relation to Figure 1. If variations in the storm electric field are to account for upward or downward motions of the ionosphere, we would expect changes in sign in the line-of-sight component of the ionospheric drift in the SAID region to be correlated with changes in sign of the Doppler shift observed in the ground scatter. Shortly after $01 \mathrm{UT}$, it is clear that the ionosphere changes from an upward motion to a downward motion, with no corresponding change in the SAID electric field.

[39] In the case of the example of Figure 3b, the perturbation appears simultaneously with the enhancement of geomagnetic activity indicated in $A L$. On the other hand, the examples of Figures $3 \mathrm{c}$ and $3 \mathrm{~d}$ appear to follow after periods of significant activity. In the latter cases, the delay suggests that the perturbations are associated with an equatorward propagating disturbance that travels at a speed of a few $100 \mathrm{~ms}^{-1}$. By way of example, if an atmospheric disturbance is produced in the vicinity of the SAID observed near range gate 60 in Figure 1, then at a speed of $500 \mathrm{~m} \mathrm{~s}^{-1}$, it will take over $1 \mathrm{~h}$ to propagate to range gate 15 , the location of the ionospheric reflection point of the leading edge of the ground backscatter. Such speeds are typical of large-scale waves (LSWs), rather than the medium-scale waves (MSWs) usually attributed to SuperDARN observations of TIDs (see inset of Figure 3a and Samson et al. [1990]). The long wavelengths of LSWs $(\sim 1000 \mathrm{~km})$ are consistent with the lack of apparent phase motion of the disturbances within the backscatter, i.e., that Doppler shifts change uniformly across the backscatter region (Figure 5). The frequencies of LSWs $(0.2$ to $0.6 \mathrm{mHz})$ are smaller than those of MSWs, and again roughly consistent with the observations of Figures $3 \mathrm{c}$ and $3 \mathrm{~d}$. Hence, we conclude that the perturbations are likely to be caused by equatorward propagating long-scale atmospheric gravity waves produced by geomagnetic disturbances in the auroral zone. Clausen et al. [2012] have demonstrated that SAIDs can persist for several hours and extend over $6 \mathrm{~h}$ of local time. Further work is required to determine the impact SAIDs can have on the atmosphere and their capability to launch waves. In this context, the lack of a delay between the observation of geomagnetic activity and the ionospheric perturbation in Figure $3 \mathrm{~b}$ is puzzling but could be due to the relatively sparse nature of the $A L$ observing network which can miss the beginning of localized activity.

[40] The magnitude of the altitude variation deduced in section $4.2, \Delta h \approx 30 \mathrm{~km}$, is large in comparison with previous studies [e.g., Samson et al., 1990; MacDougall et al., 2001], in which $\Delta h \approx 3$ to $10 \mathrm{~km}$. We expect that this increased perturbation is due to the different nature of the waves observed (LSW rather than MSW) and is also due to the nature of the WSA.

[41] We note that most previous SuperDARN observations of TIDs have concentrated on daytime phenomena, as photoionization is required to produce a significant ionosphere from which to reflect HF radio waves. In those studies, MSWs are routinely observed. In our study, the Weddell Sea Anomaly provides a reflecting ionosphere at night, and hence, nighttime TIDs are detectable. Our observations suggest that in this case, LSWs dominate. These SuperDARN observations open a new window on an otherwise poorly observed phenomenon, but further study of TIDs observed across the whole SuperDARN network is required to confirm our findings.

\section{Conclusions}

[42] The Weddell Sea Ionospheric Anomaly comprises elevated $F$ region electron densities during austral summer nights in the vicinity of the South Americas and Pacific, maintained by uplift of the ionosphere by prevailing equatorward thermospheric winds. Propagation of HF radio waves is supported at night by the WSA, as observed by the Falkland Islands SuperDARN radar. Perturbations of the WSA are observed during periods of geomagnetic activity, specifically geomagnetic storms identified in the $S Y M-H$ index. These perturbations appear to be a downward motion of the ionospheric layer followed by an upward recovery, over the course of 1 to $2 \mathrm{~h}$. The perturbations can appear singly or as trains of two to three perturbations. These perturbations are 


\section{MILAN ET AL.: TIDS IN THE WEDDELL SEA ANOMALY}

not produced by an enhancement of the prevailing equatorward thermospheric wind as this would be expected to produce uplift of the ionospheric layer. We conclude that the perturbations are associated with equatorward propagating large-scale atmospheric waves, excited by geomagnetic disturbances at auroral or subauroral latitudes. Further modeling is required to understand the nature of the waves, and in particular the downward/upward perturbation of the ionosphere.

[43] Acknowledgments. S.E.M., A.G., M.L., T.K.Y., M.P.F., G.C., and the Falkland Islands radar were supported by the Natural Environment Research Council (NERC), UK, grant NE/G019665/1. S.d.L. was supported by the United States National Science Foundation under grant AGS-0946900 to Virginia Tech. The authors acknowledge the use of SuperDARN data. SuperDARN is a collection of radars funded by national scientific funding agencies of Australia, Canada, China, France, Japan, South Africa, the United Kingdom, and the United States of America.

[44] Robert Lysak thanks Kile Baker and an anonymous reviewer for their assistance in evaluating this paper.

\section{References}

Bilitza, D. (2001), International reference ionosphere 2000, Radio Sci., 36, 261-275.

Chen, C. H., J. D. Huba, A. Saito, C. H. Lin, and J. Y. Liu (2011), Theoretical study of the ionospheric Weddell Sea Anomaly using SAMI2, J. Geophys. Res., 116, A04305, doi:10.1029/2010JA015573.

Chisham, G., et al. (2007), A decade of the Super Dual Auroral Radar Network (SuperDARN): Scientific achievements, new techniques and future directions, Surv. Geophys., 28, 33-109, doi:10.1007/s10712007-9017-8

Clausen, L. B. N., et al. (2012), Large-scale observations of a subauroral polarization stream by midlatitude SuperDARN radars: Instantaneous longitudinal velocity variations, J. Geophys. Res., 117, A05306, doi:10.1029/ 2011JA017232.

Davies, K. (1990), Ionospheric Radio, Peter Peregrinus Ltd., London.

de Larquier, S., J. M. Ruohoniemi, J. B. H. Baker, N. Ravinran Varrier, and M. Lester (2011), First observations of the midlatitude evening anomaly using
Super Dual Auroral Radar Network (SuperDARN) radars, J. Geophys. Res., 116, A10321, doi:10.1029/2011JA016787.

Greenwald, R. A., et al. (1995), DARN/SuperDARN: A global view of the dynamics of high-latitude convection, Space Sci. Rev., 71, 761-796.

Grocott, A., S. E. Milan, J. B. H. Baker, M. P. Freeman, M. Lester, and T. K. Yeoman (2011), Dynamic sub-auroral ionospheric electric fields observed by the Falkland Islands radar during the course of a geomagnetic storm, J. Geophys. Res., 116, A11202, doi:10.1029/2011JA016763.

Hall, G. E., J. W. MacDougall, D. R. Moorcroft, J.-P. St.-Maurice, A. H. Manson, and C. E. Meek (1997), Super Dual Auroral Radar Network observations of meteor echoes, J. Geophys. Res., 102, 14,603-14,614.

Hocke, K., T. Tsuda, and A. de la Torre (2002), A study of stratospheric GW fluctuations and sporadic $E$ at midlatitudes with focus on possible orographic effect of Andes, J. Geophys. Res., 107(D20), 4428, doi:10.1029/ 2001JD001330.

Holzworth, R. H., and C.-I. Meng (1975), Mathematical representation of the auroral oval, Geophys. Res. Lett., 2, 377-380.

Horvath, I. (2006), A total electron content space weather study of the nighttime Weddell Sea Anomaly or 1996/1997 southern summer with TOPEX/ Poseidon radar altimetry, J. Geophys. Res., 111, A12317, doi:10.1029/ 2006JA011679.

Horvath, I., and E. A. Essex (2003), The Weddell Sea Anomaly observed with the Topex satellite data, J. Atmos. Solar Terr. Phys., 65, 693-706, doi:10.1016/S1364-6826(03)00083-X.

MacDougall, J. W., D. A. Andre, G. J. Sofko, C.-S. Huang, and A. V. Koustov (2001), Travelling ionospheric disturbance properties deduced from Super Dual Auroral Radar measurements, Ann. Geophys., $18,1550-1559$.

Milan, S. E., T. B. Jones, T. R. Robinson, E. C. Thomas, and T. K. Yeoman (1997a), Interferometric evidence for the observation of ground backscatter originating behind the CUTLASS coherent HF radars, Ann. Geophys., 15, 29-39.

Milan, S. E., T. K. Yeoman, M. Lester, E. C. Thomas, and T. B. Jones (1997b), Initial backscatter occurrence statistics from the CUTLASS HF radars, Ann. Geophys., 15, 703-718.

Samson, J. C., R. A. Greenwald, J. M. Ruohoniemi, A. Frey, and K. B. Baker (1990), Goose Bay radar observations of Earth-reflected, atmospheric gravity waves in the high latitude ionosphere, J. Geophys. Res., 95, 7693-7709.

Wu, D. L., and J. H. Jiang (2002), MLS observations of atmospheric gravity waves over Antarctica, J. Geophys. Res., 107(D24), 4773, doi:10.1029/ 2002JD002390. 